\title{
Stage II Hilar Cholangiocarcinoma AJCC v7
}

National Cancer Institute

\section{Source}

National Cancer Institute. Stage II Hilar Cholangiocarcinoma AJCC V7. NCI Thesaurus.

Code C88060.

Stage II includes: T2a-b, N0, M0. T2a:Tumor invades beyond the wall of the bile duct to surrounding adipose tissue. T2b: T umor invades adjacent hepatic parenchyma. N0: No regional lymph node metastasis. M0: No distant metastasis. (from AJCC 7th Ed.) 